\title{
Intraoperative adverse events can be compensated by technical performance in neonates and infants after cardiac surgery: A prospective study
}

Meena Nathan, MD, ${ }^{\mathrm{a}}$ John M. Karamichalis, MD, ${ }^{\mathrm{a}}$ Hua Liu, MS, ${ }^{\mathrm{a}}$ Pedro del Nido, MD, ${ }^{\mathrm{a}}$ Frank Pigula, MD, Ravi Thiagarajan, MD, MPH, and Emile A. Bacha, MD $^{\mathrm{c}}$

Objective: Our objective was to define the relationship between surgical technical performance score, intraoperative adverse events, and major postoperative adverse events in complex pediatric cardiac repairs.

Method: Infants younger than 6 months were prospectively followed up until discharge from the hospital. Technical performance scores were graded as optimal, adequate, or inadequate based on discharge echocardiograms and need for reintervention after initial surgery. Case complexity was determined by Risk Adjustment in Congenital Heart Surgery (RACHS-1) category, and preoperative illness severity was assessed by Pediatric Risk of Mortality (PRISM) III score. Intraoperative adverse events were prospectively monitored. Outcomes were analyzed using nonparametric methods and a logistic regression model.

Results: A total of 166 patients (RACHS 4-6 [49\%]), neonates [50\%]) were observed. Sixty-one (37\%) had at least 1 intraoperative adverse event, and $47(28.3 \%)$ had at least 1 major postoperative adverse event. There was no correlation between intraoperative adverse events and RACHS, preoperative PRISM III, technical performance score, or postoperative adverse events on multivariate analysis. For the entire cohort, better technical performance score resulted in lower postoperative adverse events, lower postoperative PRISM, and lower length of stay and ventilation time $(P<.001)$. Patients requiring intraoperative revisions fared as well as patients without, provided the technical score was at least adequate.

Conclusions: In neonatal and infant open heart repairs, technical performance score is one of the main predictors of postoperative morbidity. Outcomes are not affected by intraoperative adverse events, including surgical revisions, provided technical performance score is at least adequate. (J Thorac Cardiovasc Surg 2011;142:1098-107)

Supplemental material is available online.

Outcomes after repair of complex congenital cardiac defects in infants have improved remarkably in the past 4 decades. These improvements can be attributed not only to advances in surgical techniques but also to significant developments in pediatric cardiac anesthesia, pediatric cardiopulmonary bypass (CPB), and preoperative and postoperative care in specialized intensive care units.

From the Departments of Cardiac Surgery ${ }^{\mathrm{a}}$ and Cardiology, ${ }^{\mathrm{b}}$ Children's Hospital Boston and Harvard Medical School, Boston, Mass; and Pediatric Cardiac Surgery, ${ }^{c}$ Morgan Stanley Children's Hospital of New York-Presbyterian, Columbia University College of Physicians and Surgeons, New York, NY.

Disclosures: Authors have nothing to disclose with regard to commercial support.

Read at the 91st Annual Meeting of The American Association for Thoracic Surgery, Philadelphia, Pennsylvania, May 7-11, 2011.

Received for publication May 5, 2011; revisions received July 1, 2011; accepted for publication July 11, 2011; available ahead of print Aug 16, 2011.

Address for reprints: Emile A. Bacha, MD, Columbia University College of Physicians and Surgeons, Congenital and Pediatric Cardiac Surgery, Morgan Stanley Children's Hospital of New York-Presbyterian, 3959 Broadway, CHN-274, New York, NY 10032 (E-mail: eb2709@ columbia.edu).

$0022-5223 / \$ 36.00$

Copyright (c) 2011 by The American Association for Thoracic Surgery doi:10.1016/j.jtcvs.2011.07.003
It is also widely known that outcomes, both immediate and long-term, after complex cardiac repairs in neonates and infants are dependent on multiple and interrelated factors such as case complexity, preoperative physiologic status ("severity of illness"), conduct of operation (including intraoperative anesthetic management, $\mathrm{CPB}$, intraoperative adverse events, and surgical technique), volume of cases at a given institution, and postoperative management, including the occurrence of postoperative major adverse events. ${ }^{1-4}$

Previous studies have looked at these factors in isolation or have studied a single diagnostic group or operation ${ }^{1-4}$ in both a retrospective and a prospective fashion. Other studies have looked at the impact of human factors on outcomes and concluded that human factors have an important role to play, although hidden and harder to measure, in outcomes after cardiac surgery. ${ }^{3,5}$ Previous retrospective work at our institution has focused on technical performance and established that technical performance, as measured by a technical score specific to each operation, is one of the major contributors to outcomes. ${ }^{1,4}$ We also found that optimal technical performance could neutralize the detrimental effects of poor preoperative physiology or case complexity.

The aim of this study is to prospectively define the role and relationship of each of these factors in a group of 


\section{Abbreviations and Acronyms \\ $\mathrm{CPB}=$ cardiopulmonary bypass \\ ICU = intensive care unit \\ PRISM $=$ Pediatric Risk of Mortality \\ RACHS $=$ Risk Adjustment in Congenital Heart Surgery}

neonates and infants undergoing a wide range of open cardiac repairs. Our hypothesis was that technical performance would be the most important determinant of outcomes and that technical performance would attenuate the effects of case complexity and preoperative physiologic status and neutralize the deleterious effects of intraoperative adverse events.

\section{PATIENTS AND METHODS}

Institutional review board approval was obtained for conduct of this study. Data were collected prospectively. Patients were observed intraoperatively from induction of anesthesia to transfer to the intensive care unit, and their postoperative course was followed prospectively until discharge.

\section{Data Collection}

Intraoperative observations of infants less than 6 months of age and Risk Adjustment in Congenital Heart Surgery (RACHS) categories 2 to 6 were performed from October 2008 to April 2010. All observations were performed by fully trained pediatric cardiac surgeons. All adverse events (minor or major) related to conduct of anesthesia, the operative procedure, $\mathrm{CPB}$, and events related to communication were documented. Patients of 6 surgeons (experience levels in years of practice after completion of pediatric cardiac surgery training: 1 year, 5 years, 11 years, 11 years, 20 years, and 25 years) were prospectively followed up from intraoperative observation until discharge from the hospital. Clinical data including physiologic status measurements (vital signs), laboratory tests, echocardiograms, and cardiac catheterization data were collected. Variables collected for analysis included age, weight, prematurity, presence of extracardiac and genetic abnormalities, case complexity and technical performance scores, preoperative and postoperative Pediatric Risk of Mortality (PRISM) III scores, and preoperative and postoperative lactate. The following time variables were also collected: CPB times, crossclamp times, deep hypothermic circulatory arrest and regional perfusion times (where applicable), postoperative and total intensive care unit (ICU) and hospital length of stay, and postoperative ventilation days. Need for intraoperative surgical reintervention or postoperative surgical or catheter-based reinterventions were documented.

\section{Predictor Variables}

The main predictor variables that were included in our analysis were case complexity, physiologic status, and technical performance.

Case complexity was scored on the basis of the RACHS-1 scoring system. ${ }^{6,7}$

The preoperative and postoperative physiologic status was determined using the PRISM III scoring system ${ }^{8}$ and lactate levels. For the PRISM III score, 17 physiologic variables were used. Data were collected in the 24 hours before surgery and for 48 hours after surgery. In infants who did not have preoperative arterial blood gas measured, an empiric score of 6 was given for all cyanotic lesions based on a presumed arterial oxygen tension of $40 \mathrm{~mm} \mathrm{Hg}$, and a presumptive value of 1 was assigned for lactate level. The highest lactate level in each 24-hour time period was used for scoring lactate levels.

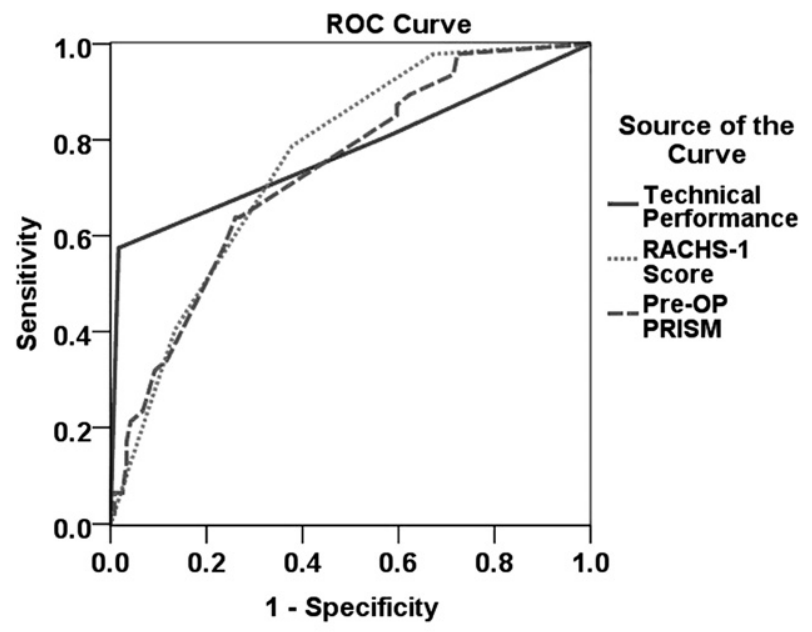

\begin{tabular}{|llll|}
\hline \multicolumn{2}{|l}{ Area Under the curve } & & \\
& Area & p Value & $95 \% \mathrm{Cl}$ \\
$\begin{array}{l}\text { Technical } \\
\text { performance }\end{array}$ & 0.774 & $<0.0001$ & $0.681-0.867$ \\
$\begin{array}{l}\text { RACHS-1 } \\
\text { Score }\end{array}$ & 0.756 & $<0.0001$ & $0.681-0.832$ \\
$\begin{array}{l}\text { Pre-Op } \\
\text { PRISM }\end{array}$ & 0.732 & $<0.0001$ & $0.650-0.814$ \\
\hline
\end{tabular}

FIGURE 1. ROC curve analysis of ability of technical performance, RACHS-1 categories, and preoperative PRISM to predict major postoperative adverse events. On logistic regression, technical performance had a higher (albeit small difference) significance in predicting occurrence of postoperative adverse events with an area under the curve of 0.774 (95\% CI, 0.681-0.867) versus 0.756 (95\% CI, 0.681-0.832) for RACHS-1 category versus 0.732 (95\% CI, 0.650-0.814). ROC, Receiver operator characteristic; RACHS, Risk Adjustment in Congenital Heart Surgery; PRISM, Pediatric Risk of Mortality; CI, confidence interval.

Technical performance was scored as previously reported from our center, ${ }^{9,10}$ based on intraoperative course, predischarge echocardiogram, catheterization data, and clinical status at discharge. In brief, each operative procedure was divided into subcategories and each subcategory was assigned an optimal, adequate, or inadequate score based on specific echocardiographic and in some instances catheterization and clinical criteria. Any intraoperative reinterventions resulted in the score being downgraded to adequate, even if the final score was "optimal." Any postoperative surgical/catheter-based reinterventions for residual defects resulted in an inadequate score. At discharge, echo and clinical status were reviewed, and for the procedure of interest, if all subcategories were optimal, then the final score was optimal; however, if one of the subcategories had an adequate score, it was scored as adequate. If any subcategory was scored as inadequate, then the final score was inadequate. Technical scores were thus graded as "optimal," "adequate," or "inadequate" for each patient for the index operation.

\section{Outcome Variables}

The outcome variables that were analyzed were the following:

- Primary: Occurrence of major postoperative adverse events

- Secondary: ventilation time, ICU length of stay, and hospital length of stay 
TABLE 1. Intraoperative surgical reintervention $(n=12 ; 7 \%)$

\begin{tabular}{llllcc}
\hline No. & RACHS & \multicolumn{1}{c}{ Diagnosis } & \multicolumn{1}{c}{ Intraoperative reintervention } & Final technical score & Outcome \\
\hline 1 & RACHS 2 & Multiple VSDs & Closure of residual and unidentified VSD & 2 & DC \\
2 & & VSD, RVOTO & Further RVOT resection & 2 & DC \\
3 & RACHS 3 & TOF/APV & Revision of RPA anastomosis & 2 & DC \\
4 & & CAVC & Repair of right AVV & 2 & DC \\
5 & & CAVC & Revision of VSD patch for AVVR & 2 & DC \\
6 & & TGA/IVS & Revision of PA anastomosis for supra systemic RV pressures & 2 & DC \\
7 & & TOF/CAVC & Further closure of LAVV cleft and further restriction of ASD & 2 & DC \\
8 & RACHS 4 & TGA/VSD & Plication of LPA to move away from left coronary button & 2 \\
9 & & TOF/MAPCAs & Fenestration of VSD & 3 & DC \\
10 & RACHS 6 & Unbalanced CAVC, & Repair AVV for residual AVVR & DC \\
& & hypoplastic arch & & Died \\
11 & & HLHC, ALCAPA & Revision of ALCAPA button & 2 \\
12 & & HLHS & Clot extraction from proximal RCA & DC \\
\hline Al
\end{tabular}

All patients who underwent intraoperative surgical reinterventions to correct residual defects identified either on transesophageal or epicardial echocardiography or based on clinical status after initial weaning from cardiopulmonary bypass. RACHS, Risk Adjustment in Congenital Heart Surgery; VSD, ventricular septal defect; $D C$, discharged; $R V O T O$, right ventricular outflow tract obstruction; $R V O T$, right ventricular outflow tract; $T O F$, tetralogy of Fallot; $A P V$, absent pulmonary valve; $R P A$, right pulmonary artery; $C A V C$, complete atrioventricular canal; $A V V$, atrioventricular valve; $A V V R$, atrioventricular valve regurgitation; TGA, transposition of the great arteries; $I V S$, intact ventricular septum; $P A$, pulmonary artery; $R V$, right ventricular; $L A V V$, left atrioventricular valve; $A S D$, atrial septal defect; $L P A$, left pulmonary artery; $M A P C A$, major aortopulmonary collateral artery; $H L H C$, hypoplastic left heart complex; $A L C A P A$, anomalous left coronary arising from pulmonary artery; $H L H S$, hypoplastic left heart syndrome; $R C A$, right coronary artery.

An adverse event was defined as "a complication that is associated with a health care intervention and is associated with suboptimal outcome." ${ }^{11} \mathrm{~A}$ major postoperative adverse event "is a complication that causes increased consumption of resources (above and beyond average), or permanent morbidity.",11

The following postoperative major adverse events were included for analysis: stroke or major neurologic deficit, need for extracorporeal membrane oxygenation, cardiac arrest requiring cardiopulmonary resuscitation, reoperation for bleeding or low cardiac output state, reoperation for residual defects, unexpected catheter-based reintervention, need for a permanent pacemaker, mediastinitis requiring surgical debridement, diaphragmatic paralysis requiring surgical plication, in-hospital mortality, or mortality in less than 30 days if discharged (per The Society of Thoracic Surgeons database criteria).

\section{Statistical Methods}

Categorical variables are summarized as numbers and percentages, and continuous variables are summarized as means \pm standard deviation with (medians and range). Patient characteristics for those with and without major postoperative adverse events were compared using the $\chi^{2}$ test for categorical variables and the Mann-Whitney test and Kruskal-Wallis test for continuous variables. Variables significant at the .05 level were considered for inclusion in a multivariable logistic regression model. Forward selection was used to fit the model. For coding "technical performance," optimal technical performance was used as reference. For coding the "RACHS- 1 categories," RACHS-1 category 2 was used as the reference. Discrimination of these factors for predicting a major postoperative adverse event was assessed using the receiver operator characteristic curve (Figure 1).

Relationships between continuous outcomes variables and continuous patient characteristics were evaluated using the Spearman rank correlation coefficient. For categorical characteristics, median values of the outcomes were compared using the Mann-Whitney test for variables with 2 categories and the Kruskal-Wallis test for variables with 3 or more categories.

A subgroup analysis was performed comparing patients who had intraoperative surgical reinterventions, patients who had postoperative surgical or catheter-based reinterventions, and those requiring no reinterventions.

IBM SPSS Statistics 18 for Window (SPSS, Inc, Chicago, Ill) was used for statistical analysis.

\section{RESULTS}

A total of 166 patients were prospectively observed and all were included in the analysis.

There were 94 (57\%) male patients. The mean age was $49.1 \pm 49.4$ days (range, $0-156$ days). Mean weight was $3.9 \pm 1.2 \mathrm{~kg}$ (range, 1.7-7.2 kg). Thirteen $(8 \%)$ weighed less than $2.5 \mathrm{~kg}$. Neonates accounted for $50 \%(\mathrm{n}=83)$. There were 10 premature infants $(<35$ weeks). Twentynine $(17 \%)$ had defined chromosomal abnormalities and $15(9 \%)$ had extracardiac abnormalities.

Eighty-four (51\%) were RACHS 2 and 3 (categorized as low risk) and $82(49 \%$ ) were RACHS 4, 5, and 6 (categorized as high risk). Diagnoses and procedures performed are represented in Tables E1 and E2.

For the entire cohort, preoperative PRISM III score was $6.3 \pm 4.3$ (mean and range, 6, 0-21), Preoperative lactate level was $1.6 \pm 1.3(1,0.6-13.2)$, CPB time was $140 \pm 59$ (132, 38-379), aortic crossclamp time was $77 \pm 38$ (75, 0-194), deep hypothermic circulatory arrest time was $22 \pm 19(12,1-75)(\mathrm{n}=57)$, and regional low-flow perfusion time was $48 \pm 18(46,5-86)(\mathrm{n}=46)$. PRISM III score on postoperative day 1 was $11.3 \pm 6.4(10,0-34)$, and lactate level on postoperative day 1 was $5.7 \pm 5.2(3.8,0-28)$. Postoperative hospital length of stay was $26 \pm 32(15$, 1-191), postoperative ICU length of stay was $15.4 \pm 24$ (8, 1-188), and postoperative ventilator time (in days) was $10 \pm 20(4,1-188)$.

Intraoperative revisions requiring reinstitution of $\mathrm{CPB}$ $(\mathrm{n}=12,7 \%)$, unexpected postoperative surgical reinterventions $(\mathrm{n}=14,11$ patients, $8 \%)$ and postoperative catheterbased reinterventions $(\mathrm{n}=22,19$ patients, $11 \%)$ are represented in Tables 1, 2, and 3. Table E3 depicts all intraoperative adverse events that were documented. Table E4 depicts 
TABLE 2. Postoperative surgical reinterventions $(n=14 ; 11$ patients; $8 \%)$

\begin{tabular}{|c|c|c|c|c|c|c|c|c|}
\hline No. & RACHS & Diagnosis & $\begin{array}{c}\text { Postop surgical } \\
\text { intervention }\end{array}$ & $\begin{array}{l}\text { Postop cath } \\
\text { intervention }\end{array}$ & $\begin{array}{l}\text { Intraop } \\
\text { revision }\end{array}$ & $\begin{array}{l}\text { Assigned tech } \\
\text { score }\end{array}$ & $\begin{array}{c}\text { Tech score based } \\
\text { on DC echo }\end{array}$ & Outcome \\
\hline 1 & 3 & $\begin{array}{l}\text { TOF/right- } \\
\text { dominant } \\
\text { CAVC, PAPVR }\end{array}$ & $\begin{array}{l}\text { Repair of recurrent } \\
\text { MR from torn } \\
\text { cleft sutures }\end{array}$ & No & No & 3 & 2 & DC home \\
\hline 2 & 3 & $\begin{array}{l}\text { HLHC: BDG, TV } \\
\text { plasty, aortic } \\
\text { valve plasty, TD } \\
\text { of RV-PA } \\
\text { conduit }\end{array}$ & $\begin{array}{l}\text { 1. TV replacement } \\
\text { 2. Repair } \\
\text { paravalvar leak } \\
\text { and } \\
\text { reconnection of } \\
\text { RV-PA conduit } \\
\text { (PPM for } \mathrm{CHB} \text { ) }\end{array}$ & No & No & 3 & 3 & Died \\
\hline 3 & 4 & $\begin{array}{l}\text { Truncus } \\
\text { arteriosus }\end{array}$ & $\begin{array}{c}\text { Revision of RV-PA } \\
\text { conduit, partial } \\
\text { closure of ASD }\end{array}$ & $\begin{array}{l}\text { BD of RV-PA } \\
\text { conduit and } \\
\text { LLPA, LLPA } \\
\text { stent }\end{array}$ & No & 3 & $\begin{array}{l}3 \text { (RVP still } \\
\text { 2/3 systemic } \\
\text { after BD) }\end{array}$ & Died \\
\hline 4 & 4 & $\begin{array}{l}\text { TOF, PA, } \\
\text { MAPCAs }\end{array}$ & $\begin{array}{l}\text { Revision of } \\
\text { unifocalized } \\
\text { RPA, banding } \\
\text { of RV-PA } \\
\text { conduit }\end{array}$ & $\begin{array}{l}\text { BD of RPA, } \\
\text { RLLPA, LPA } \\
\text { stent }\end{array}$ & No & 3 & 2 & DC home \\
\hline 5 & 4 & TGA/VSD & $\begin{array}{l}\text { Closure of } \\
\text { residual ASD }\end{array}$ & LPA stenting & No & 3 & 2 & $\mathrm{DC}$ \\
\hline 6 & 4 & $\begin{array}{l}\text { HLHC- CoA, } \\
\text { VSD, small left- } \\
\text { sided structures }\end{array}$ & $\begin{array}{l}\text { 1. Reimplantation } \\
\text { of torn AL } \\
\text { papillary } \\
\text { muscle } \\
\text { 2. MV } \\
\text { replacement } \\
\text { (PPM for CHB) }\end{array}$ & No & No & 3 & 3 & Transplantec \\
\hline 7 & 5 & Neonatal Ebstein & $\begin{array}{l}\text { RV-PA conduit, } \\
\text { TV repair and } \\
\text { repositioning }\end{array}$ & RVOT stenting & No & 3 & 2 & Died \\
\hline 8 & 6 & Shone, IAS & $\begin{array}{l}\text { Early BDG and } \\
\text { LPA plasty }\end{array}$ & $\begin{array}{c}\text { MV BD in attempt } \\
\text { to delay BDG }\end{array}$ & No & 2 & 1 & $\mathrm{DC}$ \\
\hline 9 & 6 & $\begin{array}{l}\text { UnbCAVC s/p } \\
\text { stage I, Sano, } \\
\text { AVV septation } \\
\text { for AVVR }\end{array}$ & $\begin{array}{l}\text { 1. AVV repair } \\
\text { 2. AVV repair }\end{array}$ & No & No & 3 & 3 & Died \\
\hline 10 & 6 & HLHS, ALCAPA & $\begin{array}{l}\text { Conversion of } \\
\text { BTS to RV-PA } \\
\text { conduit }\end{array}$ & No & $\begin{array}{l}\text { Revised } \\
\text { ALCAPA } \\
\text { button) }\end{array}$ & 3 & 2 & $\mathrm{DC}$ \\
\hline 11 & 6 & $\begin{array}{l}\text { d-TGA, VSD, } \\
\text { hypoplastic RV, } \\
\text { straddling TV }\end{array}$ & $\begin{array}{r}\text { TV and MV repair } \\
\text { (PPM for CHB) }\end{array}$ & $\begin{array}{c}\text { BD of innominate } \\
\text { vein, coiling } \\
\text { VV collaterals }\end{array}$ & No & 3 & 3 & $\mathrm{DC}$ \\
\hline
\end{tabular}

All patients who underwent postoperative surgical reintervention for residual defects. Note that there is higher proportion of patients in the higher RACHS-1 categories. $R A C H S$, Risk Adjustment in Congenital Heart Surgery; cath, catheter; $D C$, discharge; TOF, tetralogy of Fallot; $C A V C$, complete atrioventricular canal; $P A P V R$, partial anomalous pulmonary venous return; $M R$, mitral regurgitation; $H L H C$, hypoplastic left heart complex; $B D G$, bidirectional Glenn; $T V$, tricuspid valve; $T D$, takedown; $R V$ - $P A$, right ventricle-pulmonary artery; $P P M$, permanent pacemaker; $C H B$, complete heart block; $A S D$, atrial septal defect; $B D$, balloon dilation; $L L P A$, left lower pulmonary artery; $R V P$, right ventricular pressure; $P A$, pulmonary atresia; $M A P C A$, major aortopulmonary collateral artery; $R P A$, right pulmonary artery; $R L L P A$, right lower left pulmonary artery; $L P A$, left pulmonary artery; $T G A$, transposition of the great arteries; VSD, ventricular septal defect; $C O A$, coarctation of the aorta; $A L$, anterolateral; $R V O T$, right ventricular outflow tract; $I A S$, intra-aortic septum; $M V$, mitral valve; $U n b C A V C$, unbalanced complete atrioventricular canal; $s / p$, status post; $A V V$, atrioventricular valve; $A V V R$, atrioventricular valve regurgitation; $H L H S$, hypoplastic left heart syndrome; $A L C A P A$, anomalous left coronary arising from pulmonary artery; $d-T G A$, dextro-transposition of the great arteries; $R V$, right ventricle; $V V$, venovenous.

intraoperative reinterventions and postoperative surgical or catheter-based reinterventions based on RACHS-1 categories.

Major postoperative adverse events occurred in $47(28 \%)$ and are represented in Table E5. Thirteen $(8 \%)$ required
ECMO (1 preoperative only, 1 preoperative and postoperative, 11 postoperative).

There were $7(4.2 \%)$ deaths of which 5 occurred in neonates (Table E6). Inasmuch as the number of deaths was 
TABLE 3. Postoperative catheter interventions $(n=22 ; 19$ patients, $11 \%)$

\begin{tabular}{|c|c|c|c|c|c|c|c|c|}
\hline No. & RACHS & Diagnosis & $\begin{array}{c}\text { Postop cath } \\
\text { interventions }\end{array}$ & $\begin{array}{l}\text { Postop surgical } \\
\text { intervention }\end{array}$ & $\begin{array}{l}\text { Intraop } \\
\text { revision }\end{array}$ & $\begin{array}{l}\text { Assigned tech } \\
\text { score }\end{array}$ & $\begin{array}{l}\text { Tech score based } \\
\text { on DC echo }\end{array}$ & Outcome \\
\hline 1 & 3 & TOF, APV & BD of LUPV & No & $\begin{array}{l}\text { Revision of RPA } \\
\text { anastomosis }\end{array}$ & 2 & 2 & DC \\
\hline 2 & 3 & $\begin{array}{l}\text { LCA occlusion } \\
\text { and RV-PA } \\
\text { conduit regurg } \\
\text { s/p neonatal } \\
\text { Ross Konno }\end{array}$ & $\begin{array}{l}\text { BD of RPA } \\
\text { stenosis }\end{array}$ & No & No & 3 & 3 & $\begin{array}{l}\text { DC (required } \\
\text { OHT) }\end{array}$ \\
\hline 3 & 3 & TOF, CAVC & $\begin{array}{l}\text { ASD creation for } \\
\text { PHTN }\end{array}$ & No & No & 3 & 3 (severe PR) & DC (late death) \\
\hline 4 & 3 & $\begin{array}{l}\text { TA/PA, } \\
\text { TGA VSD, } \\
\text { s/p BTS }\end{array}$ & $\begin{array}{l}\text { BD of BTS } \\
\text { insertion and } \\
\text { branch pulm } \\
\text { arteries }\end{array}$ & No & No & 3 & $\begin{array}{l}3 \text { (2nd cath } 1 \text { mo } \\
\text { later to redialte } \\
\text { BTS and branch } \\
\text { pulm arteries }\end{array}$ & DC \\
\hline 5 & 4 & $\begin{array}{l}\text { Truncus } \\
\text { arteriosus, } \\
\text { discontinuous } \\
\text { RPA }\end{array}$ & $\begin{array}{l}\text { BD of RPA and } \\
\text { coil APC }\end{array}$ & No & No & 3 & 2 & DC \\
\hline 6 & 4 & $\begin{array}{l}\text { Truncus } \\
\text { arteriosus }\end{array}$ & $\begin{array}{l}\text { BD of RV-PA } \\
\text { conduit and } \\
\text { LLPA, LLPA } \\
\text { stent }\end{array}$ & $\begin{array}{c}\text { Revision of RV-PA } \\
\text { conduit, partial } \\
\text { closure of ASD }\end{array}$ & No & 3 & $\begin{array}{l}3 \text { (RVP still 2/3 } \\
\text { systemic after } \\
\text { BD) }\end{array}$ & Died \\
\hline 7 & 4 & TOF, MAPCAs & $\begin{array}{l}\text { BD of RPA and } \\
\text { LPA, APC } \\
\text { coiling }\end{array}$ & No & $\begin{array}{l}\text { Fenestration of } \\
\text { VSD }\end{array}$ & 3 & 3 & DC \\
\hline 8 & 4 & $\begin{array}{l}\text { TOF, PA, } \\
\text { MAPCAs }\end{array}$ & $\begin{array}{l}\text { BD of RPA, } \\
\text { RLLPA, LPA } \\
\text { stent }\end{array}$ & $\begin{array}{l}\text { Revision of } \\
\text { unifocalized } \\
\text { RPA, banding } \\
\text { of RV-PA } \\
\text { conduit }\end{array}$ & No & 3 & 2 & DC \\
\hline 9 & 4 & $\begin{array}{l}\text { TOF, PA, } \\
\text { MAPCAs, } \\
\text { premie } 34 \text { wk, } \\
\text { SGA }\end{array}$ & $\begin{array}{l}\text { 1. Bilateral pulm } \\
\text { artery dilatation } \\
\text { and stenting } \\
\text { 2. Bilateral stented } \\
\text { pulm artery } \\
\text { dilatation }\end{array}$ & No & No & 3 & 3 & DC, late death \\
\hline 10 & 4 & $\begin{array}{l}\text { d-TGA, PS, } \\
\text { hypoplastic RV }\end{array}$ & $\begin{array}{l}\text { BD of both } \\
\text { bilateral pulm } \\
\text { arteries, APC } \\
\text { coiling }\end{array}$ & No & No & 3 & 2 & DC \\
\hline 11 & 4 & TGA/VSD & LPA stenting & $\begin{array}{l}\text { Closure of residual } \\
\text { ASD }\end{array}$ & No & 3 & 2 & DC \\
\hline 12 & 4 & $\begin{array}{l}\text { TGA, VSD, arch } \\
\text { hypoplasia }\end{array}$ & $\begin{array}{l}\text { ASD dilatation } \\
\text { and LPA } \\
\text { stenting }\end{array}$ & No & No & 3 & 2 & DC \\
\hline 13 & 4 & $\begin{array}{l}\text { TGA, VSD, arch } \\
\text { hypoplasia }\end{array}$ & $\mathrm{BD}$ of RCA & No & No & 3 & 2 & DC \\
\hline 14 & 5 & Neonatal Ebstein & RVOT stenting & $\begin{array}{l}\text { RV-PA conduit } \\
\text { and TV } \\
\text { repositioning } \\
\text { and repair }\end{array}$ & No & 3 & 2 & Died \\
\hline 15 & 6 & Shone, IAS & $\begin{array}{l}\text { MV BD in } \\
\text { attempt to } \\
\text { delay BDG }\end{array}$ & $\begin{array}{l}\text { Early BDG and } \\
\text { LPA plasty }\end{array}$ & No & 2 & 1 & DC \\
\hline 16 & 6 & & & No & No & 3 & 1 & DC \\
\hline
\end{tabular}


TABLE 3. Continued

\begin{tabular}{|c|c|c|c|c|c|c|c|c|}
\hline No. & RACHS & Diagnosis & $\begin{array}{c}\text { Postop cath } \\
\text { interventions }\end{array}$ & $\begin{array}{c}\text { Postop surgical } \\
\text { intervention }\end{array}$ & $\begin{array}{l}\text { Intraop } \\
\text { revision } \\
\end{array}$ & $\begin{array}{l}\text { Assigned tech } \\
\text { score }\end{array}$ & $\begin{array}{c}\text { Tech score based } \\
\text { on DC echo }\end{array}$ & Outcome \\
\hline & & $\begin{array}{l}\text { HLHC, s/p } \\
\text { stage I BTS }\end{array}$ & $\begin{array}{l}\text { BD and stenting of } \\
\text { distal BTS and } \\
\text { ASD dilatation }\end{array}$ & & & & & \\
\hline 17 & 6 & $\begin{array}{l}\text { Heterotaxy, aortic } \\
\text { arch hypoplasia, } \\
\text { TAPVR s/p } \\
\text { DKS, arch } \\
\text { augmentation, } \\
\text { TAPVR repair }\end{array}$ & $\begin{array}{l}\text { 1. BD of pulm } \\
\text { veins } \\
\text { 2. BD of pulm } \\
\text { veins }\end{array}$ & No & No & 3 & 3 & $\mathrm{DC}$ \\
\hline 18 & 6 & HLHS, ALCAPA & $\begin{array}{l}\text { 1. Cath to stent } \\
\text { LCA and BD of } \\
\text { RPA } \\
\text { 2. Stent distal RV- } \\
\text { PA conduit }\end{array}$ & No & No & 3 & 3 & Died \\
\hline 19 & 6 & $\begin{array}{l}\text { d-TGA, VSD, } \\
\text { hypoplastic RV, } \\
\text { straddling TV }\end{array}$ & $\begin{array}{l}\text { BD of innominate } \\
\text { vein, coiling of } \\
\text { APC, TV and } \\
\text { MV repair } \\
\text { (PPM for CHB) }\end{array}$ & $\begin{array}{l}\text { TV and MV } \\
\text { repair (PPM for } \\
\text { CHB) }\end{array}$ & No & 3 & 3 & $\mathrm{DC}$ \\
\hline
\end{tabular}

All patients who underwent postoperative catheter-based reinterventions for residual defects. Note that there is a higher preponderance of patients in the higher RACHS-1 categories. RACHS, Risk Adjustment in Congenital Heart Surgery; $c a t h$, catheter; $D C$, discharge; $T O F$, tetralogy of Fallot; $A P V$, absent pulmonary valve; $B D$, balloon dilation; $L U P V$, left upper pulmonary vein; $R P A$, right pulmonary artery; $L C A$, left coronary artery; $R V-P A$, right ventricle-pulmonary artery; $s / p$, status post; $O H T$, orthotopic heart transplant; $C A V C$, complete atrioventricular canal; $A S D$, atrial septal defect; $P H T N$, pulmonary hypertension; $P R$, pulmonary regurgitation; $T A$, tricuspid atresia; $P A$, pulmonary atresia; $T G A$, transposition of the great arteries; $V S D$, ventricular septal defect; $B T S$, Blalock-Taussig shunt; $A P C$, aortopulmonary collateral artery; $L L P A$, left lower pulmonary artery; $R V P$, right ventricular pressure; $M A P C A$, major aortopulmonary collateral artery; $L P A$, left pulmonary artery; $R L L P A$, right lower left pulmonary artery; $S G A$, small for gestational age; $d$-TGA, dextro-transposition of the great arteries; $P S$, pulmonary stenosis; $R V$, right ventricle; $R C A$, right coronary artery; $T V$, tricuspid valve; $R V O T$, right ventricular outflow tract; $I A S$, intra-aortic septum; $B D G$, bidirectional Glenn; $H L H C$, hypoplastic left heart complex; TAPVR, total anomalous pulmonary venous return; $D K S$, Damus-Kaye-Stansel; $H L H S$, hypoplastic left heart syndrome; $A L C A P A$, anomalous left coronary arising from pulmonary artery; $M V$, mitral valve; $P P M$, permanent pacemaker; $C H B$, complete heart block.

small, statistical analysis of mortality as a separate outcome measure did not have enough power to correctly detect significance; therefore it was included as a component of major postoperative adverse event.

Major postoperative adverse events and postoperative ventilation times, as well as postoperative length of ICU and hospital stay, were the major and minor outcomes included in our analysis.

There was no significant difference in the rate of postoperative major adverse events or the rates of intraoperative or postoperative surgical or catheter-based reinterventions between surgeons.

On univariate analysis, higher RACHS-1 scores were associated with higher occurrence of major postoperative adverse events $(P<.0001)$, with longer ventilation days $(P<.0001)$, longer ICU lengths of stay $(P<.0001)$, and higher hospital lengths of stay $(P<.0001)$. Younger age $(P<.001)$ and lower weight $(P<.003)$ were associated with higher occurrence of major postoperative adverse events $(P<.003)$, and both were associated with higher ventilation days and postoperative ICU and hospital lengths of stay $(P<.0001)$. There was no significant difference for occurrence of major postoperative adverse events by prematurity $(P=.547)$, by presence of extracardiac or genetic abnormalities $(P=.098)$, or by need for intraoperative surgical reintervention $(P=.689)$. However, the presence of extracardiac or genetic abnormalities was associated with longer ventilation days and postoperative ICU and hospital lengths of stay $(P<.01, P<.001, P<.002$, respectively), whereas this was not significant in premature infants $(P=.745, P=.231, P=.437$, respectively). Longer median CPB times and aortic crossclamp times were associated with significantly longer ventilation times $(P, .0001$ and $P<.005$, respectively), longer postoperative ICU lengths of stay $(P<.000$ and $P<.024$, respectively), and longer postoperative hospital lengths of stay $(P<.0001$ and $P<.047$, respectively). The results of univariate analysis are included in Table 4. Bivariate association was used to choose variables with $P<.05$ for inclusion in logistic regression. Of note, prematurity, weight, neonates, and intraoperative adverse events (which included intraoperative surgical reinterventions), aortic crossclamp time, and preoperative lactate level, were found to be nonsignificant on logistic regression.

Technical performance score, RACHS category, and preoperative PRISM in combination performed best in the logistic regression analysis (Figures 1 and 2). Technical performance score, RACHS, and CPB times in combination were also significant, but slightly less powerful. 
TABLE 4. Univariate analysis

\begin{tabular}{|c|c|c|c|}
\hline \multicolumn{4}{|c|}{ Major postoperative adverse events } \\
\hline Variables & No $(n=119)$ & Yes $(n=47)$ & $P$ value \\
\hline Low birth weight $(<2.5 \mathrm{~kg})$ & $4.2 \%$ & $17 \%$ & .006 \\
\hline Prematurity & $6.7 \%$ & $4.3 \%$ & .547 \\
\hline Gender $=$ female & $46.2 \%$ & $36.2 \%$ & .239 \\
\hline Extracardiac anomaly & $6.7 \%$ & $14.9 \%$ & .098 \\
\hline Genetic anomaly & $20.2 \%$ & $10.6 \%$ & .145 \\
\hline Intraop adverse event & $28.6 \%$ & $57.4 \%$ & .001 \\
\hline $\begin{array}{l}\text { Intraop surgical } \\
\text { reintervention }\end{array}$ & $6.7 \%$ & $8.5 \%$ & .689 \\
\hline RACHS high risk & $37.8 \%$ & $78.7 \%$ & .000 \\
\hline \multicolumn{4}{|l|}{ Technical performance } \\
\hline Optimal & $42 \%$ & $19.1 \%$ & .000 \\
\hline Adequate & $56.3 \%$ & $23.4 \%$ & .000 \\
\hline Inadequate & $1.7 \%$ & $57.4 \%$ & .000 \\
\hline Neonate & $39.5 \%$ & $76.6 \%$ & .000 \\
\hline Weight & 3.9 & 3.4 & .003 \\
\hline CPB time & 123 & 176 & .000 \\
\hline Aortic crossclamp time & 72 & 81 & .047 \\
\hline $\begin{array}{l}\text { Hypothermic arrest (if } \\
\text { applicable) }\end{array}$ & 9 & 16 & .392 \\
\hline $\begin{array}{l}\text { Regional perfusion (if } \\
\text { applicable) }\end{array}$ & 42 & 48 & .290 \\
\hline Ventilation (d) & 3 & 13 & .000 \\
\hline Postop ICU length of stay & 5 & 23 & .000 \\
\hline Postop hospital length of stay & 11 & 44 & .000 \\
\hline Preop PRISM & 6 & 9 & .000 \\
\hline Preop lactate & 1 & 1.7 & .000 \\
\hline Postop day 1 PRISM & 8 & 16 & .000 \\
\hline Postop day 1 lactate & 2.6 & 8.6 & .000 \\
\hline
\end{tabular}

We performed a subgroup analysis comparing patients who had intraoperative surgical revisions with the group that required postoperative surgical or catheter-based interventions. There was a significant difference in the discharge technical score of those who had intraoperative interventions versus those who required postoperative surgical or catheter-based reinterventions $(P<.0001)$. On comparing duration of ventilation and ICU and hospital lengths of stay, there was again a significant difference $(P<.001$, $P<.001, P<.005$, respectively) (Figure 3, Figure E1). Mortality and occurrence of major postoperative adverse events was also significantly different (Figure E2).

\section{DISCUSSION}

This study demonstrates that, across a broad spectrum of complex neonatal and infant cardiac repairs, technical performance score had the strongest association with outcomes. In addition, it points to what can be called a "technical imperative," an absolute rule whereby it is "imperative" to leave the operating room with a good or

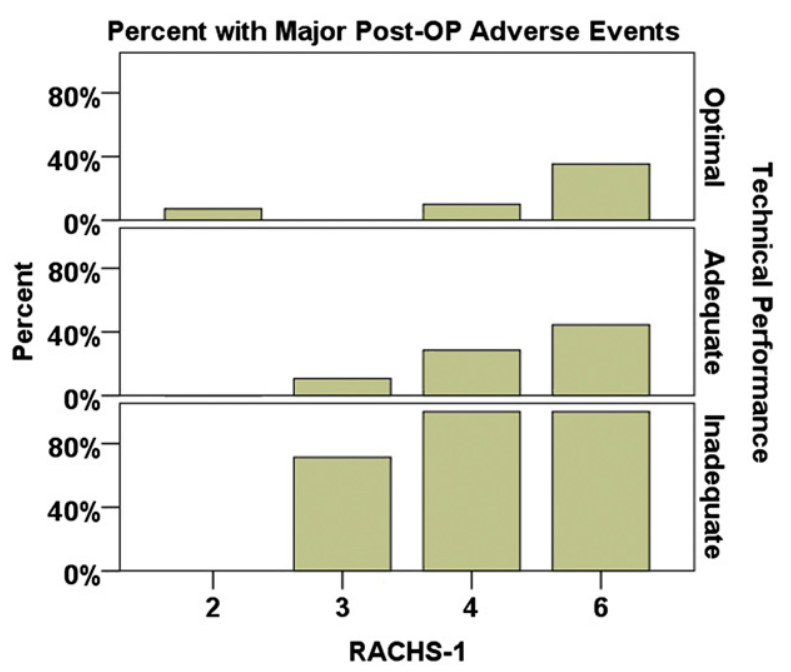

FIGURE 2. Major postoperative adverse events based on technical scores and RACHS-1 categories. This figure outlines the percentage of patients with major postoperative adverse events comparing technical performance and RACHS-1 risk categories. Optimal technical performance had a low occurrence of adverse events, whereas adequate technical performance had low rates of adverse events in the low complexity groups (RACHS-1 categories 2 and 3, but higher percentage of adverse events in the higher risk categories (RACHS-1 categories 4 and 6). Inadequate technical performance had the highest percentage with adverse events in all categories of the RACHS-1 system. RACHS, Risk Adjustment in Congenital Heart Surgery.

better yet optimal technical result, even if this requires surgical revision. Indeed, the patients who required intraoperative revisions did not do significantly worse than the patients who had no intraoperative and no postoperative reinterventions. On the other hand, failure to detect and intervene on intraoperative findings of inadequate technical result led to delayed intervention, either surgical or catheter-based, with its resultant increase in occurrence of postoperative adverse events, prolonged lengths of stay and ventilation time, as well as mortality.

It is obvious that surgical outcomes are not solely dependent on the technical prowess of the surgeon. This study also showed that the RACHS-1 score, a risk stratification paradigm currently most used by insurance companies, performed almost as well as the technical score in predicting outcomes, followed closely by the preoperative PRISM score, an index of physiologic illness severity. Whereas the RACHS- 1 class is a fixed value that cannot be modified, the physiologic status of an infant can often be improved by medical means before surgical repair. This therefore also vindicates another surgical dogma, namely, to not operate on an infant if there is a possibility of improving the infant's physiologic status. ${ }^{12}$

This study is a single-center study and looks at cases by 6 surgeons of vastly different experience levels. We did not restrict ourselves to a single procedure or diagnosis. Thus, this 


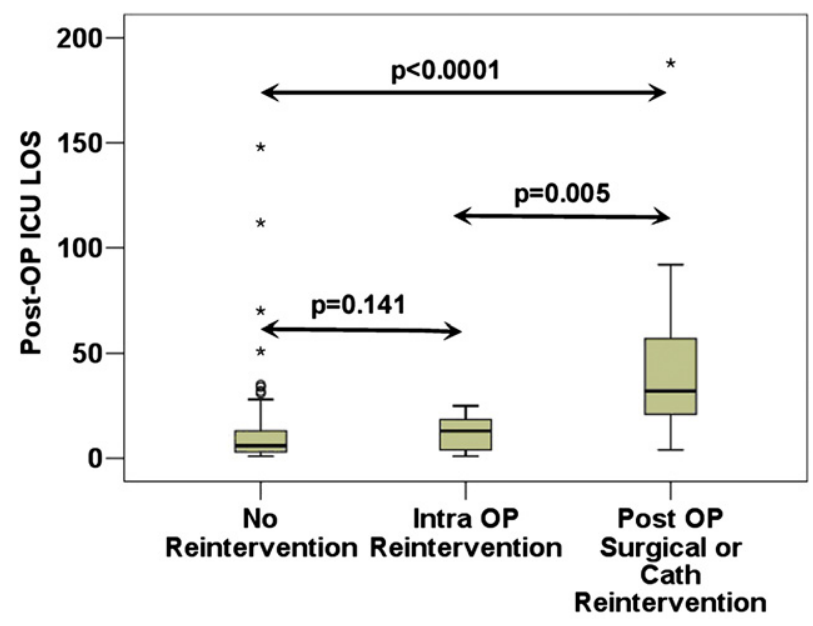

FIGURE 3. Postoperative ICU LOS for intraoperative surgical reinterventions versus postoperative surgical reinterventions or catheter-based reinterventions when compared with those who had no intervention: The was no statistical difference in the median LOS for patients who had no reinterventions ( 6 days) versus patients with intraoperative reinterventions (13 days) $(P=.141)$. However, patients who had postoperative surgical or catheter-based reinterventions had a significantly longer LOS (32 days) $(P=.005$ vs intraoperative reinterventions, $P<.0001$ vs no reinterventions). *Outliers. ICU, Intensive care unit; LOS, length of stay.

prospective study is likely to be as good a representative snapshot as possible from a large pediatric heart center. Furthermore, all observers were trained pediatric cardiac surgeons, increasing the quality of the observed intraoperative data, and minimizing the likelihood of missing an important event. Previous work from our institution had shown the importance of technical performance in outcomes, specifically for the Norwood stage I procedure where it had a direct impact on hospital survival. Optimal technical performance also attenuated the effects of case complexity and physiologic severity of illness. ${ }^{1}$ A similar effect (postoperative morbidity escalated by worse physiologic status, but attenuated by improved surgical technique) was also shown for another high-risk surgical procedure, namely, pancreatic resection, in a prospective study of 412 patients. ${ }^{13}$

For "routine" cases of complete atrioventricular canal, tetralogy of Fallot, and ventricular septal defect repairs, as well as arterial switches, in part owing to the very low mortality seen with these procedures, the impact of technical scores on mortality was not as dramatic. Many researchers have therefore switched to indices of morbidity,${ }^{14}$ such as the occurrence of major postoperative events as used in the present study. The study's focus on complex infant repairs further improved our "yield." In accordance with many other studies, we found that a substantial number of infants have significant adverse events during the postoperative period. ${ }^{15,16}$ Defining and addressing the factors that contribute to those is a logical way to improve patient outcomes and also decrease health care cost.
Pediatric cardiac surgery outcomes often depend on the function of fragile microstructures. Thus, it is not surprising that a certain number of patients require intraoperative revision. This study is the first to compare patients who underwent intraoperative revisions with those who required postoperative interventions. The results are unequivocal and show that it is the end result that counts. Technical compensation was able to neutralize the deleterious effects of having to go back on CPB a second time. $\mathrm{CPB}$ time came in as a close fourth behind technical scores, RACHS-1, and PRISM III scores for inclusion in the logistic regression analysis. Longer time was significantly associated with a higher incidence of major postoperative adverse events, a finding that is not surprising given the known deleterious effects of prolonged CPB times, especially in neonates. However, we do not think that there is a proven cause and effect relationship in this respect, and our study supports the fact that technical outcome is by far more important than shortening CPB time.

Study limitations include the fact that this is a singlecenter study, with results that may not be generalizable to other centers. The numbers in each risk category are relatively small, inasmuch as a wide range of diagnoses was included. The PRISM score has not been validated for cyanotic heart disease, but there is currently no accepted way to measure preoperative illness severity.

In conclusion, this prospective study involving $166 \mathrm{com}-$ plex neonatal and infant cardiac repairs shows that optimal technical performance scores result in best outcomes, even if an intraoperative revision has to be performed, and attenuates the effects of poor preoperative physiology across a wide spectrum of diagnoses and procedures.

\section{References}

1. Karamichalis JM, Thiagarajan RR, Liu H, Mamic P, Gauvreau K, Bacha EA Stage I Norwood: optimal technical performance improves outcomes irrespective of preoperative physiological status or case complexity. J Thorac Cardiovasc Surg. 2010;139:962-8.

2. de Leval MR, Carthey J, Wright DJ, Farewell VT, Reason JT. Human factors and cardiac surgery: a multicenter study. J Thorac Cardiovasc Surg. 2000;119: 661-72.

3. Barach P, Johnson JK, Ahmad A, Galvan C, Bognar A, Duncan R, et al. A prospective observational study of human factors, adverse events, and patient outcomes in surgery for pediatric cardiac disease. $J$ Thorac Cardiovasc Surg. 2008; $136: 1422-8$.

4. Karamichalis JM, del Nido PJ, Thiagarajan RR, Liu H, Jenkins KJ, Liu H, et al. Early postoperative severity of illness predicts outcomes after the stage I Norwood procedure. Ann Thorac Surg. 2011 Jun 23 [Epub ahead of print].

5. Schraagen JM, Schouten T, Smit M, Hass F, van der Beek D, van den Ven J, et al A prospective study of paediatric cardaic surgical Microsystems: assessing the relationships between non routine events, team work and patient outcomes. BMJ Qual Saf. 2011;20:599-603. Epub 2011 Apr 13.

6. Jenkins KJ, Gauvreau K, Newburger JW, Spray TL, Moller JH, Iezzoni LI. Consensus-based method for risk adjustment for surgery for congenital heart disease. J Thorac Cardiovasc Surg. 2002;123:110-8.

7. Jenkins KJ, Gauvreau K. Center-specific difference in mortality: preliminary analyses using the Risk Ajustment in Congenital Surgery (RACHS-1) method. J Thorac Cardiovasc Surg. 2002;124:97-104.

8. Pollack MM, Patel KM, Ruttimann UE. PRISM III: an updated pediatric risk of mortality score. Crit Care Med. 1996;24:743-52. 
9. Larrazabal LA, del Nido PJ, Jenkins KJ, Gauvreau K, Lacro R, Colan SD, et al. Measurement of technical performance in congenital heart surgery: a pilot study. Ann Thorac Surg. 2007;83:179-84.

10. Bacha EA, Larrazabal LA, Pigula FA, Jenkins KJ, Gauvreau K, Colan SD, et al. Measurement of technical performance in surgery for congenital heart disease: the stage I Norwood procedure. J Thorac Cardiovasc Surg. 2008;136:993-7.

11. Jacob JP, Benavidez OJ, Bacha EA, Walters HL, Jacob ML. The nomenclature of safety and quality of care for patients with congenital cardiac disease: a report of the Society of Thoracic Surgeons Congenital Database Taskforce Subcommittee on Patient Safety. Cardiol Young. 2008;18(suppl. 2):81-91.

12. Vincent C, Moorthy K, Sarker SK, Chang A, Darzi AW. Systems approaches to surgical quality and safety. From concept to measurement. Ann Surg. 2004;239: 474-82.

13. Pratt W, Callery MP, Vollmer CM. Optimal surgical performance attenuates physiological risk in high-acuity operation. J Am Coll Surg. 2008;207:717-30.

14. Welke KF, Karamlou T, Ungerleider RM, Diggs BS. Mortality rate is not a valid indicator of quality differences between pediatric cardiac surgical programs. Ann Thorac Surg. 2010;89:139-46.

15. Benavidez OJ, Gauvreau K, Bacha E, del Nido P, Jenkins KJ. Application of a complication screening method to congenital heart surgery admissions. Pediatr Cardiol. 2008;29:258-65.

16. Benavidez OJ, Gauvreau K, del Nido P, Bacha E, Jenkins KJ. Complications and risk factors for mortality during congenital heart surgery admissions. Ann Thorac Surg. 2007;84:147-55.

\section{Discussion}

Dr Marc R. de Leval (London, United Kingdom). I congratulate Dr Nathan for this very clear presentation. This work is an addition to the already impressive list of contributions of Dr Bacha and colleagues to outcome analysis, risk management, and patient safety research. In a previous study, they had introduced the concept of technical performance as measured by technical scores specific to each operation. The technical scores are graded as optimal, adequate, and inadequate.

In this study, they have undertaken a prospective analysis of the impact of technical performance on outcomes for the whole spectrum of congenital heart defects in infants in a single institution, the operations being performed by 6 different surgeons at various stages of their training and experience. The RACHS scoring system was used for the case complexity and the PRISM scoring system was used to score the preoperative and postoperative physiologic status.

The findings were as follows:

1. Technical performance is the single most important determinant of outcomes.

2. Optimal technical performance mitigates the effects of case complexity and physiologic severity of the illness.

3. Optimal technical performance leads to good outcomes even if an intraoperative revision is required. The technical compensation neutralizes the deleterious effects of having to go back on $\mathrm{CPB}$ a second time.

4. Intraoperative revisions lead to better results than postoperative interventions.

5. The physiologic status of the patient can often be improved by medical means before surgery and this improves the overall results.

I have 1 comment and 3 questions. I find the term "technical performance" somewhat misleading. It gives the impression that it relates essentially to surgical dexterity. I personally believe that going back on CPB and revising successfully a complex repair in a sick infant requires as much cognitive skills as technical skills. There is some analogy within sports psychology where studies of physical, technical, and mental skills show that the only predictor of final Olympic achievements, the difference between gold and silver medalists, was the mental readiness, which is made of commitment, confidence, and positive thinking. What you have actually analyzed is the quality of the repair rather than technical performance. What do you think about this comment? This is my first question.

The aviation industry has introduced crew resource management training to manage adverse events. Do you think this could be applicable to surgery by the so-called nontechnical skills technique training, and do you think that this could be introduced in the curriculum of medical schools and residency programs? This is my second question.

The operations were performed by 6 surgeons. Was there a difference in the outcomes of those 6 surgeons?

My last point is a minor point. You do not discuss in the manuscript what kind of investigations were done intraoperatively to assess the quality of the repair. I assume that assessments were made by transesophageal echocardiogram. I wonder whether the reintervention rate intraoperatively could be increased by refining the intraoperative assessment of your repair, thus reducing the need for postoperative interventions.

Dr Nathan. Thank you, Dr de Leval, for your questions. I agree that the term "technical performance" seems to implicate the surgeons, whereas outcomes in surgery for congenital cardiac disease are not only dependent on the way the surgery is performed but on several of other factors, including the postoperative course and ICU care. We have therefore decided to refine our statement and call this technical performance scores rather than just technical performance.

I agree that the airline industry has excellent simulation programs, and I think there are several centers beginning simulation programs, including our center, for management of events in the operating room.

There were 6 surgeons involved in this study, and there was no significant difference among the technical performance scores of these surgeons.

We did use intraoperative transesophageal echocardiography for the bigger babies, and if the babies were really small, we used epicardial echocardiography. We are in the process of defining criteria for each of these methods of investigation and are also developing a technical performance score for intraoperative assessment.

Dr Muhammad Mumtaz (Norfolk, Va). I have 2 questions. Do I understand from your conclusions that if the outcome in the operating room is less than optimal, revising the result to make it the ideal outcome does not affect any adverse events? That is to say, if one goes back on for a second pump run on the pump and revises, whether it was a residual ventricular septal defect or whatever, then it does not affect how the baby is going to do? Do I understand that correctly?

Dr Nathan. Yes, you do.

Dr Mumtaz. It sounds a little bit odd. I would just intuitively disagree with that.

Second, I want to focus on the premature babies. There are 10 noted in your series, and you said you defined them by less than 
35 weeks at birth. At what age were these babies offered surgery? Were these offered surgery at less than 35 weeks of corrected age or were they offered surgery at a different corrected age?

Dr Nathan. The majority of them are operated on in the first week of life; unless their diagnosis warranted surgery at 2 to 3 months, as is the case with tetralogy of Fallot and complete atrioventricular canal defects, which are operated on a few weeks later.
Dr Mumtaz. So at your institution, the protocol for premature babies does not consider the corrected age? Do I understand that correctly?

Dr Nathan. It depends on the complexity of the case and need for intervention. We did not use prematurity as an indicator to postpone surgery for any length of time if the case was complex and needed immediate surgery.

Dr Mumtaz. Thank you. 
Post-Operative Adverse Events

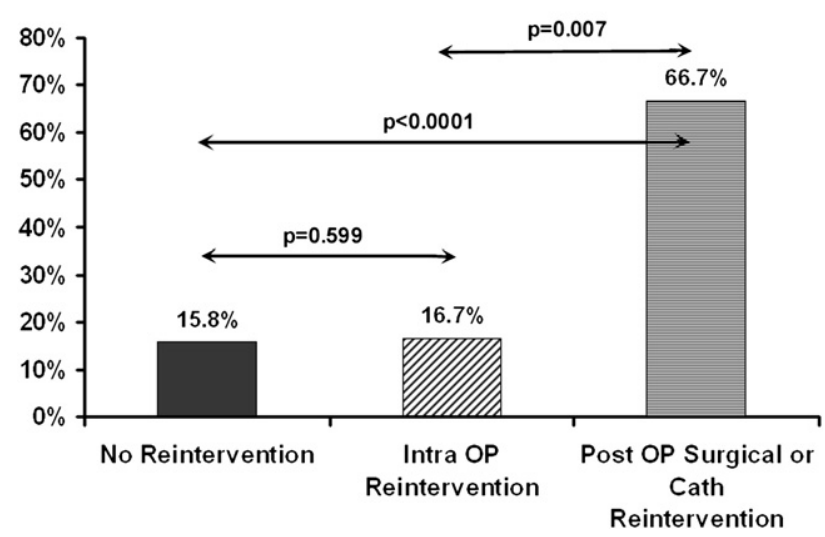

FIGURE E1. Comparison of occurrence of major adverse events for no intervention versus intraoperative intervention versus postoperative surgical or catheter-based interventions. There was significantly higher incidence of major postoperative adverse events in the postoperative surgical/catheter reinterventions group $(P<.0001$ vs no intervention, $P=.007$ intraoperative surgical reinterventions).
Mortality

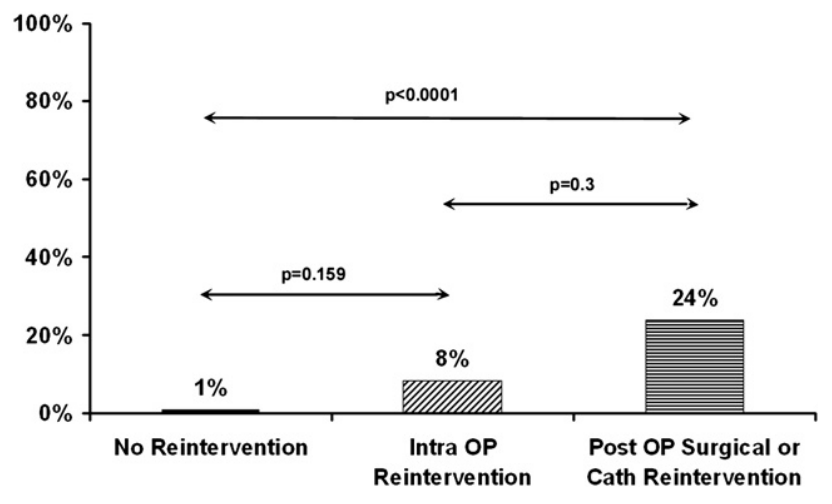

FIGURE E2. Comparison of mortality for no intervention versus intraoperative intervention versus postoperative surgical or catheter-based interventions. There is a significantly higher mortality for patients who underwent postoperative surgical/catheter reinterventions when compared with those who had no reintervention $(P<.0001)$. 
TABLE E1. List of diagnoses in 166 patients

\begin{tabular}{|c|c|c|}
\hline $\mathrm{VSD}=15$ & Truncus arteriosus $=6$ & $\mathrm{DORV}=3$ \\
\hline $\mathrm{TOF} / \mathrm{PS}=19$ & $\mathrm{AP}$ window $=2$ & DOLV $=1$ \\
\hline $\begin{array}{l}\text { TOF/PA/single } \\
\text { ductlike } \\
\text { collateral = } 2\end{array}$ & TGA/IVS $=7$ & $\begin{array}{l}\text { Malposed atrial } \\
\quad \text { septum }=1\end{array}$ \\
\hline $\begin{array}{l}\text { TOF/PA/ } \\
\quad \text { MAPCAs }=6\end{array}$ & TGA/VSD $=9$ & LV-aorta tunnel $=1$ \\
\hline $\mathrm{TOF} / \mathrm{APV}=2$ & Tricuspid atresia $=4$ & $\mathrm{AS} / \mathrm{AR}=5$ \\
\hline $\mathrm{TOF} / \mathrm{CAVC}=4$ & HLHS $=24$ & $\mathrm{MR}=1$ \\
\hline $\mathrm{CAVC}=13$ & $\mathrm{HLHC}=9$ & $\mathrm{PA} / \mathrm{IVS}=2$ \\
\hline Transitional AVC $=2$ & Neonatal TAPVR $=4$ & Supra AS/PS = 1 \\
\hline $\begin{array}{l}\text { CoA, arch } \\
\quad \text { hypoplasia }=1\end{array}$ & $\mathrm{UCAVC}=4$ & Neonatal Ebstein $=1$ \\
\hline $\mathrm{IAA}=3$ & DILV $=1$ & \\
\hline $\mathrm{CoA} / \mathrm{VSD}=5$ & $\begin{array}{l}\text { Heterotaxy/ } \\
\text { UnbCAVC/ } \\
\text { TAPVR, etc }=8\end{array}$ & \\
\hline
\end{tabular}

$\overline{V S D}$, Ventricular septal defect; TOF, tetralogy of Fallot; $P S$, pulmonary stenosis; $P A$, pulmonary atresia; $M A P C A$, major aortopulmonary collateral artery; $A P V$, anomalous pulmonary vein; $C A V C$, complete atrioventricular canal; $A V C$, atrioventricular connection; $C O A$, coarctation of the aorta; $I A A$, interrupted aortic arch; $A P$, aortopulmonary; TGA, transposition of the great arteries; IVS, interventricular septum; $H L H S$, hypoplastic left heart syndrome; $H L H C$, hypoplastic left heart complex; $T A P V R$, total anomalous pulmonary venous return; UCAVC, unbalanced complete atrio-ventricular canal; DILV, double-inlet left ventricle; UnbCAVC, unbalanced complete atrioventricular canal; DORV, double-outlet right ventricle; DOLV, double-outlet left ventricle; $L V$, left ventricle; $A S$, aortic stenosis; $A R$, aortic regurgitation; $M R$, mitral regurgitation.

TABLE E2. List of all procedures performed based on RACHS-1 categories $(n=166)$

\begin{tabular}{lll}
\hline RACHS $2(\mathbf{n}=\mathbf{4 0})$ & TV repair $=2$ & TOF/PA full repair with unifocalization $=5$ \\
AP window repair $=2$ & CAVC $=13$ & IAA/VSD $=2$ \\
Common atrial septation $=1$ & RMBTS $=2$ & Rastelli $=3$ \\
TOF, TAP $=2$ & TAPVR $=1$ & Neonatal Ross Konno $=2$ \\
TOF valve sparing $=15$ & ASO $=7$ & ASO/VSD $=8($ with arch $=5)$ \\
RVOT patch $=1$ & Transitional AVC $=1$ & Arch augmentation/pulmonar artery band $=2$ \\
BDG $=3$ & Aortic valve repair $=2$ & Biventricular repair for Shone complex $=2$ \\
Kawashima $=1$ & Valve sparing root replacement $=1$ & CoA + arch $=1$ \\
VSD $=15$ & Supra AS/PS repair $=1$ & RACHS $\mathbf{5}(\mathbf{n}=\mathbf{1}) *$ \\
RACHS $\mathbf{3}(\mathbf{n}=\mathbf{4 4})$ & LV-aorta tunnel $=1$ & Neonatal Ebstein's repair $=1$ \\
DORV/VSD baffle $=2$ & RACHS $\mathbf{4}(\mathbf{n}=\mathbf{4 7})$ & RACHS $\mathbf{6}(\mathbf{n}=\mathbf{3 4})$ \\
MV repair $=2$ & Neonatal TAPVR $=5$ & Norwood BTS $=9$ \\
TOF/APV or pulmonary artery conduit $=2$ & VSD + arch $=6$ & Norwood Sano $=21$ \\
Conduit change $=2$ & Truncus arteriosus repair $=6$ & DKS $=4$ \\
TOF/CAVC $=4$ & Comprehensive stage II $=2$ & \\
TOF supramitral ring $=1$ & Unifocalization only $=3$ & \\
\hline
\end{tabular}

RACHS, Risk Adjustment in Congenital Heart Surgery; $A P$, aortopulmonary; TOF, tetralogy of Fallot; TAP, transannular patch; RVOT, right ventricular outflow tract; $B D G$, bidirectional Glenn; $V S D$, ventricular septal defect; $D O R V$, double-outlet right ventricle; $M V$, mitral valve; $A P V$, absent pulmonary valve; $C A V C$, complete atrioventricular canal; $T V$, tricuspid valve; RMBTS, Blalock-Taussig shunt; TAPVR, total anomalous pulmonary venous return; $A S O$, arterial switch operation; $A V C$, atrioventricular canal; $A S$, aortic stenosis; $P S$, pulmonary stenosis; $L V$, left ventricle; $I A A$, interrupted aortic arch; $C o A$, coarctation of the aorta; $D K S$, Damus-Kaye-Stansel. *The single patient in RACHS-1 category 5 was included in category 6 for analysis. 
TABLE E3. Intraoperative adverse events patient $(n=61 ; 37 \%)$

Main problem area

Bleeding (17)

Bleeding intraoperatively $=9$ (cannula site, ductus arteriosus, descending thoracic aorta)

Reexploration for bleeding $=7$ (chest initially closed in 3 )

Factor VII for uncontrolled ongoing bleeding $=1$

Perfusion/CPB

Venous line fell on floor during circulatory arrest $=1$

Air in oxygenator secondary to failure of low level indicator $=1$ (successfully picked up and purged—no patient adverse event)

Inadvertent drainage of patient during $\mathrm{CPB}$ wean (new machine) $=1$

Manifold leak in circuit requiring change $=1$

High membrane pressure, high filling pressures on ECMO $=1$

Lower body malperfusion $=1$

Inadvertent venous decannulation $=1$

Air in venous line from failure to clamp $=2$

Air lock in venous line $=1$

Airway/pulmonary

Difficulty in ventilation from abnormal lung compliance $=2$

Difficult intubation $=1$

Arrhythmias

SVT requiring cardioversion $=3$

$\mathrm{VT} / \mathrm{VF}$ requiring cardioversion $=5$

$\mathrm{CHB} / \mathrm{JR} / \mathrm{EAT}$ requiring pacing $=16$

SVT requiring urgent cannulation $=1$

Technical

Reinstitution of CPB for revision of residual defects $=12$

Inadvertent inclusion of left main in suture to control bleeding from RV-PA conduit (recognized and suture removed) $=1$

LPA dissection $=1$

Reinitiation of CPB to repair SVC cannulation $=1$

ST changes from air in coronary $=1$

ST changes after ALCAPA repair $=2$ (1 revised in operating room, 1 required LCA stent $)$

Pulmonary valve tear after $\mathrm{BD}$ requiring repair $=3$ ( 1 required conversion to TAP)

Miscellaneous

Cardiac arrest $=5$ (pre-CPB arrest $=3$, post-CPB arrest urgent cannulation $=2$ )

Reinstitution of CPB for period of rest for LCOS, RV hypertension, hyperkalemia, air embolus, arrhythmia, LV distention $=8$

Hypotension during epicardial echo $=1$

Post-CPB arrest, CPR, cardioversion $=2$

Difficulty in CVL access $=1$

Chest reopened for LCOS, arrhythmia $=5$

ST changes in RVDCC requiring higher perfusion pressures $=1$

ECMO for inability to wean off $\mathrm{CPB}=3$

Clipping of BTS for pulmonary overcirculation $=1$

Delay in arrival of blood products $=1$

Intraoperative adverse events: This includes events related to anesthesia, operative procedure, cardiopulmonary bypass, and events related to communication (some patients had more than 1 event). $C P B$, Cardiopulmonary bypass; $E C M O$, extracorporeal membrane oxygenation; $S V T$, supraventricular tachycardia; $V T$, ventricular tachycardia; $V F$, ventricular fibrillation; $C H B$, complete heart block; $J R$, junctional rhythm; $E A T$, ectopic atrial tachycardia; $R V-P A$, right ventricle-pulmonary artery; $L P A$, left pulmonary artery; $S V C$, superior vena cava; $A L C A P A$, anomalous left coronary arising from pulmonary artery; $L C A$, left coronary artery; $B D$, balloon dilation; $T A P$, transannular patch; $L C O S$, low cardiac output state; $R V$, right ventricle; $L V$, left ventricle; $C P R$, cardiopulmonary resuscitation; $C V L$, central venous line; $R V D C C$, right ventricle-dependent coronary circulation; $B T S$, Blalock-Taussig shunt. 
TABLE E4. Rates of reinterventions based on RACHS-1 categories

\begin{tabular}{|c|c|c|c|c|c|c|c|c|}
\hline $\begin{array}{l}\text { RACHS-1 } \\
\text { categories }\end{array}$ & $\begin{array}{c}\text { Intraop } \\
\text { reinterventions }\end{array}$ & $\begin{array}{c}\text { Postop } \\
\text { surgical } \\
\text { reinterventions }\end{array}$ & $\begin{array}{c}\text { Postop } \\
\text { catheter-based } \\
\text { reinterventions }\end{array}$ & $\begin{array}{c}\text { Median } \\
\text { ventilation } \\
\text { time (d) } \\
\end{array}$ & $\begin{array}{c}\text { Median } \\
\text { postop } \\
\text { ICU } \\
\text { LOS } \\
\text { (d) } \\
\end{array}$ & $\begin{array}{c}\text { Median } \\
\text { postop } \\
\text { hospital } \\
\text { LOS } \\
\text { (d) } \\
\end{array}$ & $\begin{array}{c}\text { Major } \\
\text { postop } \\
\text { adverse } \\
\text { events } \\
\end{array}$ & Mortality \\
\hline $\begin{array}{l}\text { RACHS-1 category } 2 \\
\quad(\mathrm{n}=40)\end{array}$ & $5 \%$ & $0 \%$ & $0 \%$ & 1 & 3 & 6 & $2.5 \%$ & $0 \%$ \\
\hline $\begin{array}{l}\text { RACHS- } 1 \text { category } 3 \\
\quad(n=44)\end{array}$ & $11.4 \%$ & $4.6 \%$ & $9.1 \%$ & 3 & 6 & 12 & $20.5 \%$ & $2.3 \%$ \\
\hline $\begin{array}{l}\text { RACHS- } 1 \text { category } 4 \\
\quad(\mathrm{n}=47)\end{array}$ & $4.3 \%$ & $10.6 \%$ & $21.3 \%$ & 7 & 11 & 21 & $38.3 \%$ & $6.4 \%$ \\
\hline $\begin{array}{l}\text { RACHS- } 1 \text { category } 6 \\
\quad(\mathrm{n}=35)\end{array}$ & $8.6 \%$ & $11.4 \%$ & $14.3 \%$ & 10 & 17 & 29 & $54.3 \%$ & $8.6 \%$ \\
\hline
\end{tabular}

TABLE E5. List of all major postoperative adverse events $(n=47$;

$28 \%$ )

Neurologic $=10$

Subdural hemorrhage $=1$

Intraventricular hemorrhage $=3$

Cerebrovascular accidents $=4$

Seizures from small vessel emboli $=2$

Reexploration for bleeding $=12$

Reexploration for hemodynamic issues $=16$

Low cardiac output state $=10$

Restrictive right ventricular physiology $=1$

Hypoxia $=1$

Cardiac arrest $=1$

Blalock-Taussig shunt/Sano clipping $=2$

Removal of clip from shunt $=1$

Unbanding of right ventricle-pulmonary artery conduit $=1$

Cardiac arrest/unstable rhythm $=3$

Cardiopulmonary resuscitation $=1$

Cardioversion $=1$

Pericardiocentesis for tamponade $=1$

Postoperative extracorporeal membrane oxygenation $=12$

Postoperative surgical reinterventions for residual defects $=12$

Postoperative catheter reinterventions for residual defects $=18$

Complete heart block requiring intervention $=5$

Diaphragm plication $=1$

Sternal debridement $=2$

Miscellaneous $=2$

Fasciotomy for lower extremity ischemia $=1$

Exploration of retroperitoneal hematoma $=1$

Some patients had more than 1 event. 
TABLE E6. Mortality data $(n=7 ; 4.2 \%)$

\begin{tabular}{|c|c|c|c|c|c|}
\hline & Diagnosis (age) & $\begin{array}{l}\text { RACHS-1 } \\
\text { category }\end{array}$ & $\begin{array}{l}\text { Technical } \\
\text { score }\end{array}$ & Cath reintervention & Surgical reintervention \\
\hline 1 & $\begin{array}{l}\text { HLHS, BDG, TV repair, aortic } \\
\text { valvotomy }(100 \mathrm{~d})\end{array}$ & 3 & 3 & 0 & $\begin{array}{l}\text { Reop-TVR } \\
\text { Reop-repair of paravalvular leak }\end{array}$ \\
\hline 2 & TOF/PA/MAPCAs (96 d) & 4 & 2 & 0 & 0 \\
\hline 3 & Truncus arteriosus $(3 \mathrm{~d})$ & 4 & 3 & BD of RV-PA conduit and LLPA & $\begin{array}{l}\text { Revision of RV-PA conduit and clot } \\
\text { extraction, ASD restriction }\end{array}$ \\
\hline 4 & Neonatal Ebstein $(1 \mathrm{~d})$ & 5 & 3 & RVOT stenting & Reop RV-PA conduit and TV repair \\
\hline 5 & $\begin{array}{l}\text { Heterotaxy, AA, CAVC, severe } \\
\text { AVVR (5 d) }\end{array}$ & 6 & 3 & 0 & Reoperation $\times 2$ to repair AVVR \\
\hline 6 & $\begin{array}{l}\text { UnbCAVC, arch hypoplasia, } \\
\text { AVVR ( } 23 \mathrm{~d})\end{array}$ & 6 & 3 & 0 & 0 \\
\hline 7 & $\begin{array}{l}\text { HLHC, dysplastic MV, } \\
\text { ALCAPA }(2 \mathrm{~d})\end{array}$ & 6 & 3 & $\begin{array}{l}2 \text { (a) Stenting of LCA and RPA dilatation; } \\
\text { (b) RPA dilatation; stenting of RV-PA } \\
\text { conduit }\end{array}$ & 0 \\
\hline
\end{tabular}

